\title{
A rare case of gestational thyrotoxicosis as a cause of acute myocardial infarction
}

\author{
Varalaxmi Bhavani Nannaka and Dmitry Lvovsky \\ Division of Pulmonary and Critical Care Medicine, Bronx Lebanon Hospital Center, Bronx, New York, USA
}

Correspondence should be addressed to $V$ B Nannaka

Email

vnannka@bronxleb.org

\section{Summary}

Angina pectoris in pregnancy is unusual and Prinzmetal's angina is much rarer. It accounts for $2 \%$ of all cases of angina. It is caused by vasospasm, but the mechanism of spasm is unknown but has been linked with hyperthyroidism in some studies. Patients with thyrotoxicosis-induced acute myocardial infarction are unusual and almost all reported cases have been associated with Graves' disease. Human chorionic gonadotropin hormone-induced hyperthyroidism occurs in about $1.4 \%$ of pregnant women, mostly when hCG levels are above $70-80000 \mathrm{IU} / \mathrm{L}$. Gestational transient thyrotoxicosis is transient and generally resolves spontaneously in the latter half of pregnancy, and specific antithyroid treatment is not required. Treatment with calcium channel blockers or nitrates reduces spasm in most of these patients. Overall, the prognosis for hyperthyroidism-associated coronary vasospasm is good. We describe a very rare case of an acute myocardial infarction in a 27-year-old female, at 9 weeks of gestation due to right coronary artery spasm secondary to gestational hyperthyroidism with free thyroxine of $7.7 \mathrm{ng} / \mathrm{dL}$ and TSH $<0.07 \mathrm{IU} / \mathrm{L}$.

\section{Learning points:}

- AMI and cardiac arrest due to GTT despite optimal medical therapy is extremely rare.

- Gestational hyperthyroidism should be considered in pregnant patients presenting with ACS-like symptoms especially in the setting of hyperemesis gravidarum.

- Our case highlights the need for increased awareness of general medical community that GTT can lead to significant cardiac events. Novel methods of controlling GTT as well as medical interventions like ICD need further study.

\section{Background}

Acute myocardial infarction (AMI) in women during childbearing age is uncommon. Pregnancy has been shown to increase the risk of AMI by 3 to 4 times from that of general population $(1,2)$. The estimated occurrence of AMI during pregnancy is $3-10$ cases per 100000 deliveries with an overall maternal mortality rate of $21 \%$ and fetal mortality rate of $13 \%$ (1). Prinzmetal's angina is very rare, and it accounts for $2 \%$ of all cases of angina. Prinzmetal's angina commonly occurs at rest. People with Prinzmetal's angina are frequently younger than those with the other forms of angina. It is caused by vasospasm, the mechanism of which is unidentified, but have been linked with hyperthyroidism in some studies (3).

The first case of spontaneous coronary vasospasm documented on angiography in a hyperthyroid patient was reported in 1979 (4). Patients with thyrotoxicosisinduced AMI are rare and almost all reported cases have been associated with Graves' disease.

We describe a very rare case of an acute myocardial infarction in a 27-year-old female at 9 weeks of gestation due to right coronary artery spasm secondary to gestational hyperthyroidism. 


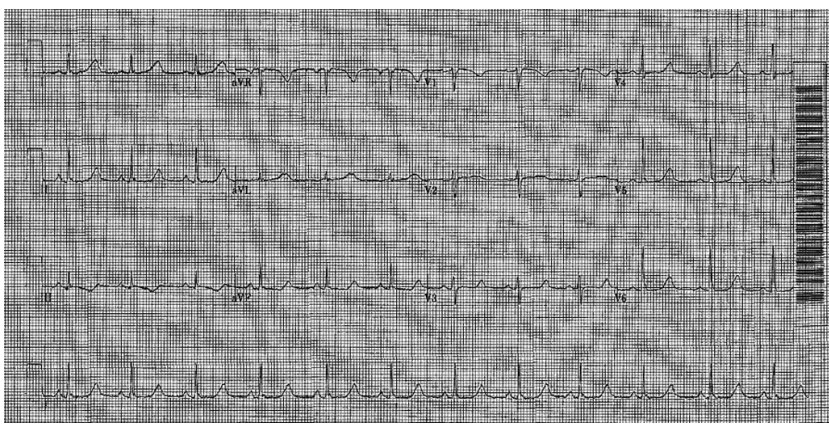

Figure 1

EKG at the time of presentation.

\section{Case presentation}

A 27-year-old 9-week pregnant woman presented to emergency room with the complaint of 3 weeks of generalized weakness, nausea and vomiting. Patient also reported two weeks of chest discomfort, radiating to the back, not associated with shortness of breath, decrease in exercise tolerance, dizziness, blurry vision or loss of consciousness. She could not recall any exacerbating or relieving factors. She denied history of fever, palpitations, chills, rashes, cough, diarrhea or dysuria. There was no history of travel or food poisoning. There was no history of hypertension, diabetes, coronary artery disease or any other medical problems. She denied

Table 1 Laboratory parameters on admission.

\begin{tabular}{l} 
Parameters \\
\hline Hemoglobin $(\mathrm{g} / \mathrm{dL})$ \\
Hematocrit $(\%)$ \\
Platelet count $(\mathrm{k} / \mu \mathrm{L})$ \\
Leucocyte count $(\mathrm{k} / \mu \mathrm{L})$ \\
Serum sodium $(\mathrm{mEg} / \mathrm{L})$ \\
Serum potassium $(\mathrm{mEq} / \mathrm{L})$ \\
Serum chloride (mEq/L) \\
Serum bicarbonate $(\mathrm{mEq} / \mathrm{L})$ \\
BUN (mg/dL) \\
Creatinine $(\mathrm{mg} / \mathrm{dL})$ \\
Serum glucose $(\mathrm{mg} / \mathrm{dL})$ \\
Total Protein $(\mathrm{g} / \mathrm{dL})$ \\
Serum albumin $(\mathrm{g} / \mathrm{dL})$ \\
Aspartate transaminase (unit/L) \\
Alanine aminotransferase (unit/L) \\
Alkaline phosphatase (unit/L) \\
Total bilirubin (mg/dL) \\
Direct bilirubin (mg/dL) \\
Lactic acid level (mmol/L) \\
Urine analysis \\
Blood cultures \\
Urine cultures \\
Urine toxicology
\end{tabular}

\begin{tabular}{c}
\hline Values \\
\hline 15.4 \\
47 \\
127 \\
13.6 \\
133 \\
2.4 \\
77 \\
30 \\
43 \\
1.1 \\
151 \\
8.5 \\
4.8 \\
285 \\
549 \\
97 \\
6.1 \\
4.5 \\
2.6 \\
Negative \\
Negative \\
Negative \\
Cannabinoids
\end{tabular}

http://www.edmcasereports.com smoking tobacco but admitted smoking marijuana. Her medication history only included prenatal vitamins.

On physical examination, she was afebrile, the heart rate was 79 beats per minute, the respiratory rate was 16 breaths per minute, her blood pressure was 122/55 $\mathrm{mmHg}$ and oxygen saturation was 98\% on room air. Her mucous membranes were dry, and cardiopulmonary and abdominal examination was unremarkable. There was no pedal edema or calf tenderness present.

\section{Investigation}

Initial electrocardiogram (EKG) showed normal sinus rhythm with ventricular rate of 72 beats per minute (Fig. 1). Laboratory results revealed mild hyponatremia with serum sodium of $133 \mathrm{mEq} / \mathrm{L}$, severe hypokalemia of $2.4 \mathrm{mEq} / \mathrm{L}$, metabolic alkalosis with serum bicarbonate of $30 \mathrm{mEq} / \mathrm{L}$ and serum $\mathrm{pH}$ of 7.59. Liver function tests showed transaminitis and hyperbilirubinemia. Urine toxicology was positive for cannabinoids (Table 1). Patient was admitted to medical intensive care unit with the initial impression of hyperemesis gravidarum resulting in electrolyte abnormalities. Twelve hours after initial presentation, patient complained of worsening chest discomfort radiating to the back. Repeat EKG revealed $3 \mathrm{~mm}$ ST segment elevation in leads II, III and AVF (Figs 2 and 3), and was started on Heparin drip, b-blockers, aspirin and statin. Probability of pulmonary embolic disease as well as aortic and coronary dissection were considered and effectively ruled out by computed tomography with contrast. Cardiac markers showed troponin $\mathrm{T}$ from $1.410 \mathrm{ng} / \mathrm{mL}$ peaking at $3.600 \mathrm{ng} / \mathrm{mL}$ after $12 \mathrm{~h}$ (Table 2). Pelvic and transvaginal ultrasound was suggestive of normal gestational size fetus without abnormalities. Other laboratory results revealed low thyroid-stimulating hormone level (TSH) of $<0.07 \mathrm{IU} / \mathrm{L}$; T4 $24.8 \mu \mathrm{g} / \mathrm{dL}$, T3 $377 \mathrm{ng} / \mathrm{dL}$; and free thyroxine of $7.77 \mathrm{ng} / \mathrm{dL}$ suggesting hyperthyroid state (Table 2). Graves' disease was ruled out with negative thyroid-stimulating immunoglobulin and anti-thyroid peroxidase antibodies (Table 2). Serum erythrocyte sedimentation rate was normal. Patient was diagnosed with gestational hyperthyroidism.

\section{Treatment}

She was started on dexamethasone and loaded with iodine to achieve euthyroid state. In addition, patient 


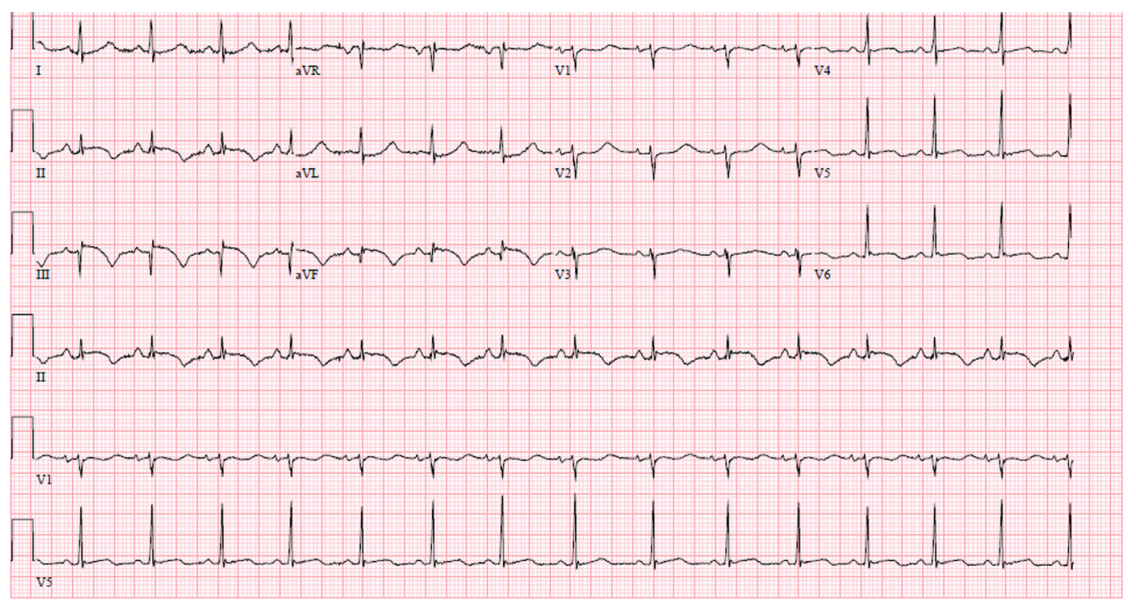

\section{Figure 2}

EKg prior to coronary angiography. underwent coronary angiography, which showed severe right coronary artery spasm (Fig. 4) with improvement after administration of intra-coronary nitroglycerine (Fig. 5). A diagnosis of Prinzmetal angina was established, and she was started on calcium channel blocker. Patient chose to terminate the pregnancy. After $24 \mathrm{~h}$, the patient went into episodes of non-sustained ventricular tachycardia (NSVT) and sustained (SVT) ventricular tachycardia, which degenerated into ventricular fibrillation (VF) with conversion to normal sinus rhythm after single electrical cardioversion. She was intubated and placed on mechanical ventilation during the advanced cardiac life support protocol. Later during the hospital course, she had recurrent episodes of NSVT and SVT with Torsade De Pointes (Fig. 6) and VF despite amiodarone and lidocaine therapy. Patient was then transferred to a facility where she had an intra-cardiac defibrillator placement. Subsequently, she was liberated from mechanical ventilation and discharged home.

\section{Outcome and follow-up}

Patient was then transferred to a facility where she had an intra-cardiac defibrillator placement. Subsequently, she was liberated from mechanical ventilation and discharged home on calcium channel blockers and antiarrhythmic agent. A month after her discharge from the hospital, she was seen in an endocrinology and cardiology clinic for follow-up.

\section{Discussion}

Gestational transient thyrotoxicosis (GTT) of nonautoimmune origin is caused by the stimulation of the TSH receptor through human chorionic gonadotropin hormone (5). Human chorionic gonadotropin hormone-induced hyperthyroidism occurs in about $1.4 \%$ of pregnant women, mostly when hCG levels are above 70-80000 IU/L (6). GTT is characterized by an elevated serum thyroid hormone level and low or

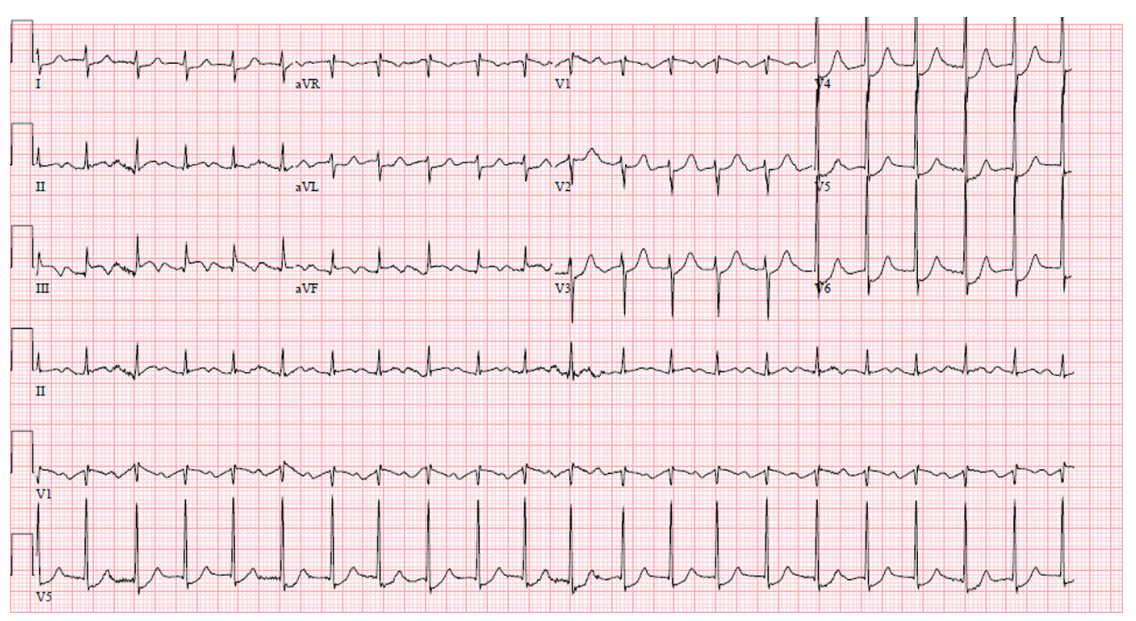

Figure 3

EKG two days after coronary angiography. ECG showed ST elevation in the inferior leads and ST depression in anterolateral leads. 


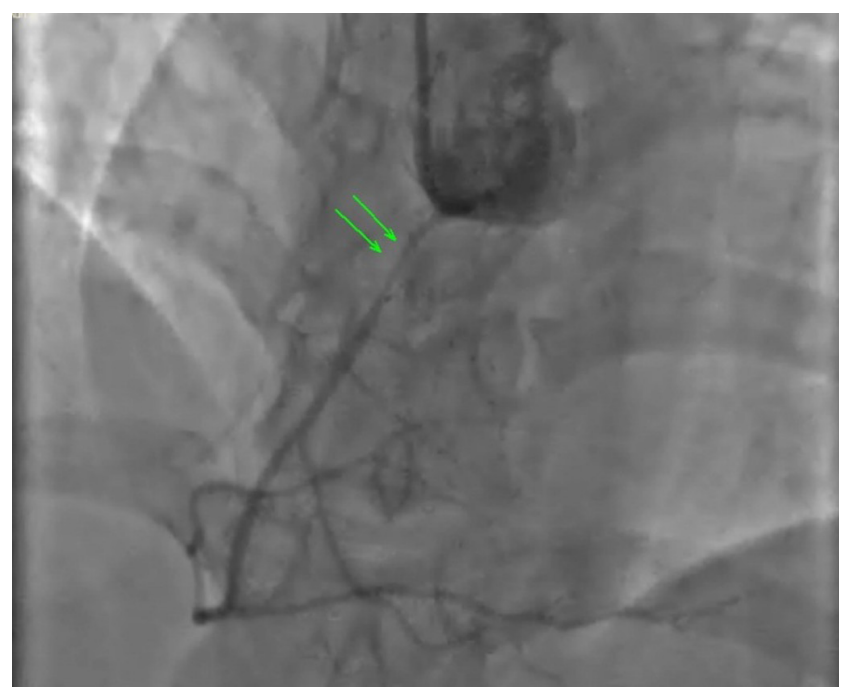

Figure 4

Cardiac catheterization showing severe proximal RCA spasm.

undetectable serum TSH level in the absence of TSH receptor antibody. GTT has been associated with hyperemesis gravidarum. Hyperemesis gravidarum occurs in about $1-3 \%$ of pregnancies. Circulating free $\mathrm{T}_{4}$ and free $\mathrm{T}_{3}$ levels were often elevated in hyperemetic patients suggesting an association between the severity of the hyperemesis and the laboratory evidence of hyperthyroidism. On the other hand, the hCG levels in women with hyperemesis compared to those without emesis are similar; however, the former has augmented thyroid-stimulating activity. Kimura et al. hypothesized

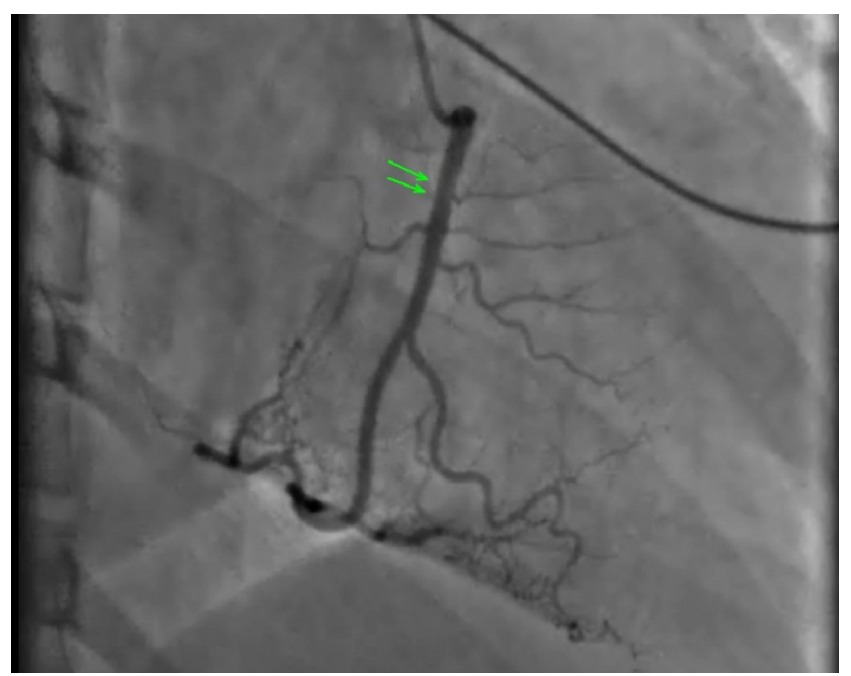

Figure 5

Cardiac catheterization showing severe proximal RCA spasm with improvement after intra-coronary nitroglycerine infusion.

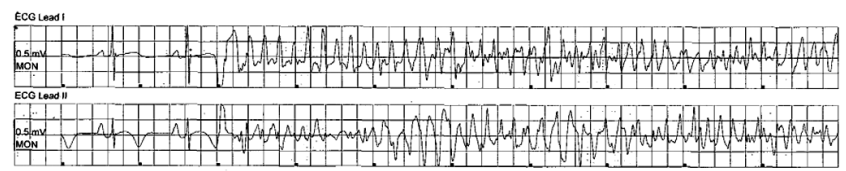

Figure 6

Torsade pointes.

that the hyperemetic patients produce molecular variants of hCG with increased thyroid-stimulating activity (7). Women with gestational thyrotoxicosis may be distinguished from those with Graves' disease by the lack of goiter and the absence of antithyroid antibodies. GTT is transient and typically resolves spontaneously in the latter half of pregnancy, and specific antithyroid treatment is usually not required. However, it was not the case in the patient described previously.

Thyroid hormones have a direct effect on cardiac myocytes, endothelial cells and vascular smooth muscle cells, resulting in hypertrophy and stiffness of the vessels (8). Napoli et al. studied peripheral arterial reactivity in patients with hyperthyroidism before and after treatment (9). They confirmed that although nitric oxide mediates basal vasodilatation in untreated hyperthyroidism, the vasoconstrictor response to norepinephrine was significantly increased in untreated hyperthyroidism compared to the response in control subjects and in those effectively treated. Consequently, coronary vasospasm in hyperthyroidism might result from an amplified sensitivity to norepinephrine and/or a diminished response to nitric oxide-mediated vasodilatation in the coronary arteries. The mechanism(s) responsible for this discordant response leading to vasospasm remains unknown.

Review of literature reveals case reports of hyperthyroidism-associated coronary vasospasm have described different presentations, including angina ischemia, myocardial infarction, cardiogenic shock,

Table 2 Cardiac markers and thyroid panel during the admission.

\begin{tabular}{|c|c|c|c|c|}
\hline & Day 1 & Day 1 & Day 2 & Day 2 \\
\hline Troponin T (ng/mL) & 1.410 & 3.190 & 3.600 & 3.110 \\
\hline Creatine kinase $(\mu / L)$ & 1107 & 2236 & 2971 & 1344 \\
\hline Creatine kinase MB $(n g / m L)$ & 66.59 & 81 & & \\
\hline TSH (IU/L) & $<0.07$ & $<0.07$ & $<0.07$ & $<0.07$ \\
\hline T3 (ng/dL) & 377 & 298 & 169 & 149 \\
\hline $\mathrm{T} 4$ (ug/dL) & $>24.8$ & 22.8 & & \\
\hline Free thyroxin (ng/dL) & 7.77 & 7.74 & 3.72 & 2.89 \\
\hline
\end{tabular}


cardiac arrest with death, ventricular arrhythmias and pulmonary vasospasm mimicking pulmonary embolism. Coronary artery spasm frequently causes lifethreatening ventricular arrhythmias and sudden cardiac death. Criteria for diagnosis of AMI in pregnant women are the same as those in non-pregnant patients and comprise primarily of symptoms, electrocardiographic changes and cardiac markers. However, the diagnostic approach is influenced by physiological changes during pregnancy and fetal safety. Our patient experienced AMI and potentially fatal arrhythmias resulting in multiple cardiac arrests secondary to gestational hyperthyroidism, which is a very unusual presentation and has not been reported in the literature to the best of our knowledge.

In general, the prognosis for hyperthyroidismassociated coronary vasospasm is good. Nitrates and calcium channel blockers may be needed to avoid recurrent episodes. Ultimately, radioiodine ablation or thyroidectomy might be indicated for patients with refractory disease. Although implantable cardioverter defibrillators (ICD) have improved mortality in high-risk patients with coronary artery spasm, the efficacy of ICD in patients with ventricular fibrillation (VF) associated to coronary artery spasm is still debatable (10). Our patient eventually had an ICD placement for recurrent ventricular fibrillation, which makes our case unique because GTT is usually transient and mild, but in the case we are describing, hyperthyroid state was refractory to medical therapy.

\section{Declaration of interest}

The authors declare that there is no conflict of interest that could be perceived as prejudicing the impartiality of the research reported.

\section{Funding}

This research did not receive any specific grants from any funding agency in the public, commercial or not-for-profit sector.
Patient consent

Written informed consent was obtained from the patient.

\section{Author contribution statement}

Varalaxmi Nannaka searched the literature and wrote the paper. Dmitry Lvovsky supervised the patient treatment and critically revised and edited the paper. All authors have made significant contributions to the paper and have reviewed it before submission. All authors have read and approved the final paper.

\section{References}

1 Roth A \& Elkayam U 1996 Acute myocardial infarction associated with pregnancy. Annals of Internal Medicine 125 751-762. (doi:10.7326/0003-4819-125-9-199611010-00009)

2 James AH, Jamison MG, Biswas MS, Brancazio LR, Swamy GK \& Myers ER 2006 Acute myocardial infarction in pregnancy: a United States population-based study. Circulation 113 1564-1571. (doi:10.1161/CIRCULATIONAHA.105.576751)

3 Masani ND, Northridge DB \& Hall RJ 1995 Severe coronary vasospasm associated with hyperthyroidism causing myocardial infarction. British Heart Journal 74 700-701. (doi:10.1136/hrt.74.6.700)

4 Wei JY, Genecin A, Greene HL \& Achuff SC 1979 Coronary spasm with ventricular fibrillation during thyrotoxicosis: response to attaining euthyroid state. American Journal of Cardiology 43 335-339. (doi:10.1016/S0002-9149(79)80023-5)

5 Glinoer D, De Nayer P, Robyn C, Lejeune B, Kinthaert J \& Meuris S 1993 Serum levels of intact human chorionic gonadotropin (HCG) and its free alpha and beta subunits, in relation to maternal thyroid stimulation during normal pregnancy. Journal of Endocrinological Investigation 16 881-888. (doi:10.1007/BF03348950)

6 Glinoer D 1998 Thyroid hyperfunction during pregnancy. Thyroid 8 859-864. (doi:10.1089/thy.1998.8.859)

7 Kimura M, Amino N, Tamaki H, Ito E, Mitsuda N, Miyai K \& Tanizawa O 1993 Gestational thyrotoxicosis and hyperemesis gravidarum: possible role of hCG with higher stimulating activity. Clinical Endocrinology 38 345-350. (doi:10.1111/j.1365-2265.1993.tb00512.x)

8 Owen PJ, Sabit R \& Lazarus JH 2007 Thyroid disease and vascular function. Thyroid 17 519-524. (doi:10.1089/thy.2007.0051)

9 Napoli R, Biondi B, Guardasole V, Matarazzo M, Pardo F, Angelini V, Fazio S \& Saccà L 2001 Impact of hyperthyroidism and its correction on vascular reactivity in humans. Circulation 104 3076-3080. (doi:10.1161/hc5001.100621)

10 Meisel SR, Mazur A, Chetboun I, Epshtein M, Canetti M, Gallimidi J, Katz A, Strasberg B \& Peled B 2002 Usefulness of implantable cardioverter-defibrillators in refractory variant angina pectoris complicated by ventricular fibrillation in patients with angiographically normal coronary arteries. American Journal of Cardiology 89 1114-1116. (doi:10.1016/S0002-9149(02)02283-X)

Received in final form 7 September 2016 Accepted 25 October 2016 